\title{
Zur Freizeitnutzung von Schweizer Wäldern: ihre Bedeutung für die regionale Ökonomie
}

Luisa Vogt

Marco Pütz
Eidgenössische Forschungsanstalt für Wald, Schnee und Landschaft (CH), Fachhochschule Südwestfalen, Soest (DE)*

Eidgenössische Forschungsanstalt für Wald, Schnee und Landschaft $(\mathrm{CH})$

\section{Recreational use of Swiss forests: their importance for the regional economy}

\begin{abstract}
Forests fulfill conservation, production and welfare functions. If forests are not considered isolatedly, but rather as part of a landscape or a region, their positive impact on regional welfare becomes apparent. The regional economic impact of the recreational use of Swiss forests is investigated in the case study regions of Sihlwald, a typical local recreation area, and Bergell, a destination for nature-based tourism. The relative importance of forests in the decision to visit the case study regions is identified by means of a conjoint analysis that assesses the forest affinity of visitors. Both the regional added value and the employment effects of the recreational use of forests are identified in an economic impact analysis. The results show that although they have a high affinity for forests, visitors to local recreation forests, such as the Sihlwald, spend little and generate rather small regional economic impacts. In contrast, the findings for nature-based tourism destinations, such as the Bergell, show a different picture: although the forest is not the most important criterion when selecting the destination, the regional economy benefits from higher consumer expenditures. The amount of visitor expenditure most notably depends on the variety and quality of tourism services in and around the forest area. Hence, to promote the regional economic importance of the recreational use of forests, tourism services in forest areas would have to be created or enhanced.
\end{abstract}

Keywords: forest recreation, forest tourism, Switzerland, regional economic impact doi: 10.3188./szf.2011.0396

* Lübecker Ring 2, DE-59494 Soest, E-Mail vogt@fh-swf.de

W älder erfüllen verschiedene Funktionen beziehungsweise Leistungen. Sie bieten Schutz vor Naturgefahren, produzieren Holz, bieten Lebensraum für Tiere und Pflanzen, sind Freizeit- und Erholungsraum für Menschen und liefern weitere Leistungen für die Allgemeinheit wie zum Beispiel saubere Luft. Diese Schutz-, Nutz- und Wohlfahrtsfunktionen sind im Bundesgesetz vom 4. Oktober 1991 über den Wald (Waldgesetz, WaG, SR 921.0) verankert und charakterisieren die Multifunktionalität des Ökosystems Wald. Wird der Wald nicht isoliert, sondern als Teil der Landschaft oder als Teil einer Region betrachtet, werden zudem die positiven Effekte des Waldes für die regionale Wohlfahrt deutlich. Dieser Beitrag untersucht die regionalökonomische Bedeutung der Freizeitnutzung von Schweizer Wäldern. Die regionalökonomische Perspektive auf den Wald ist konzeptionell verwandt mit der ökonomischen Dienstleistungsperspektive und der integrativen Landschaftssystemperspektive, wie sie von Olschewski \& Elsasser (2010) vorgeschla- gen werden. Der Vorteil dieser Perspektiven besteht darin, die naturräumlichen und wirtschaftlichen Verflechtungen von Wäldern mit ihrer Umgebung explizit in der Analyse berücksichtigen zu können.

Bei der Bewertung von Ökosystemleistungen von Wäldern steht zumeist die Quantifizierung des gesamtgesellschaftlichen Nutzens im Fokus. Grundsätzlich gilt, dass sich der Wert von Ökosystemleistungen nur zum Teil direkt in Wirtschaftskreisläufen wiederfindet, so zum Beispiel im Fall privater Güter wie Holz. Handelt es sich bei den Leistungen jedoch um öffentliche Güter (Mankiw 2004), fehlt ein Markt mit Preisen, die als Indikatoren für den Wert gelten können. Die gesellschaftliche Wertschätzung von Waldleistungen lässt sich damit nur indirekt, zum Beispiel über Zahlungsbereitschaftsanalysen, erfassen. Ein solches öffentliches Gut eines Waldes stellt oft die kulturelle Leistung «Erholung» dar (Millennium Ecosystem Assessment 2005), denn der freie Zutritt zu Wäldern ist in Mitteleuropa meist gesetzlich garantiert. Trotz des Gemeingutcha- 
rakters können Erholungsleistungen von Wäldern und die forstwirtschaftlichen Leistungen zur Erholungsvorsorge aber monetäre Kreisläufe auslösen. Zum Beispiel können die Waldgäste im Zusammenhang mit ihrem Waldbesuch touristische Angebote im Umfeld des Waldes nutzen und dafür bezahlen. Dieser regionalökonomische Wert von Wäldern ist nach Kenntnis der Autoren bisher kaum untersucht.

Der folgende Beitrag untersucht am Beispiel des Sihlwalds als eines typischen Naherholungsgebiets und des Bergells als naturtouristischer Ferienregion die regionalökonomische Bedeutung der Freizeitnutzung von Schweizer Wäldern. Methodisch finden eine Wertschöpfungsanalyse Verwendung sowie eine Conjoint-Analyse zur Bestimmung des Anteils der «waldaffinen» Waldbesucher am Total der Gäste, für welche primär der Wald und nicht zum Beispiel eine bestimmte Freizeitaktivität ausschlaggebend für den Waldbesuch ist.

\section{Methodisches Vorgehen}

\section{Wertschöpfungsanalyse}

Der Beitrag eines Wirtschaftszweigs oder Tourismussegments zur regionalen Wertschöpfung lässt sich über verschiedene Ansätze ermitteln (Mayer et al 2010). In der Schweiz und in Deutschland haben sich im Kontext des Naturtourismus Wertschöpfungsstudien etabliert, welche die durch die Ausga-

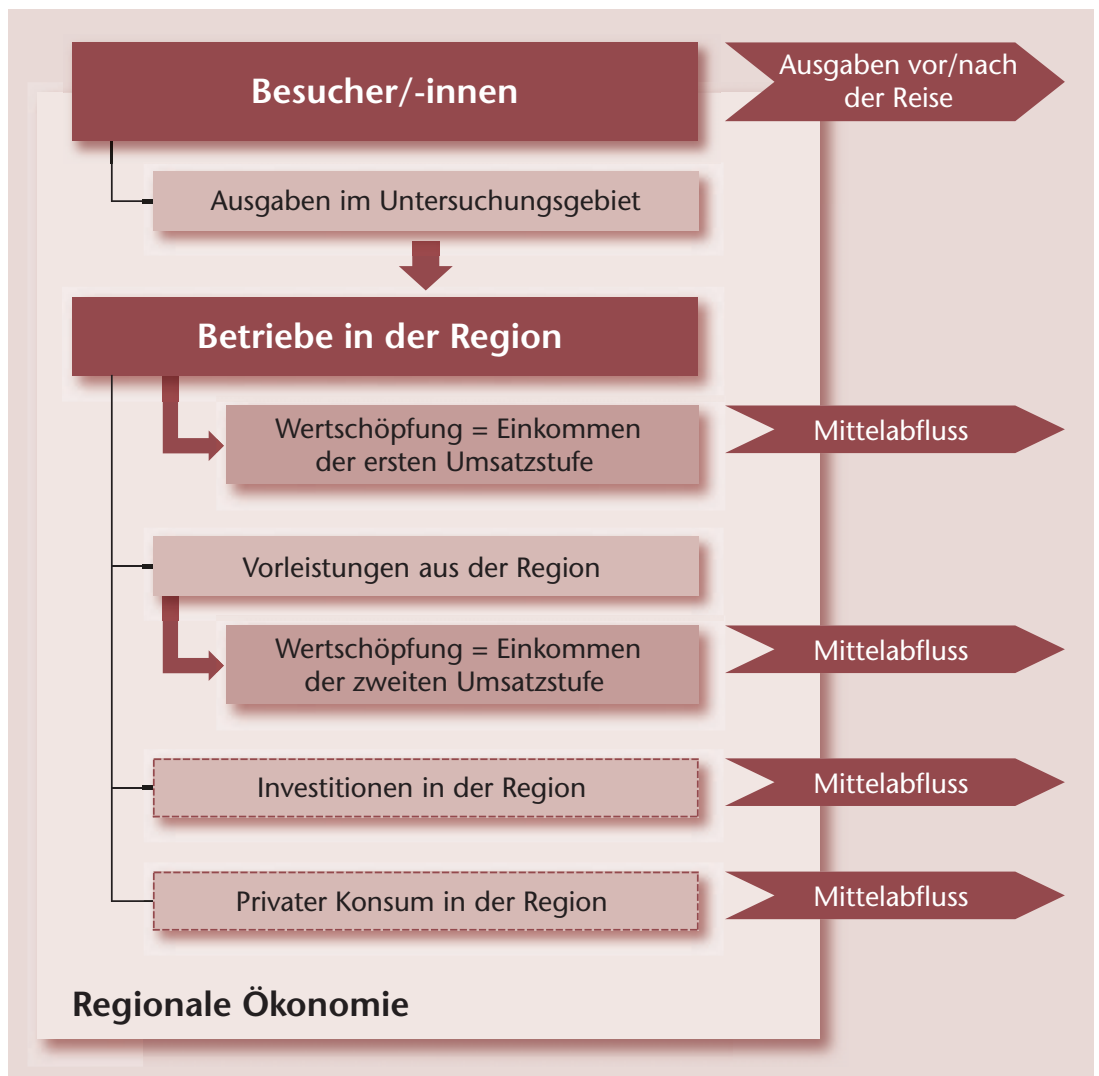

Abb 1 Methodisches Vorgehen bei einer Wertschöpfungsanalyse (Quelle: Metzler 2007, verändert). ben der Besucher ausgelöste Wertschöpfung fokussieren (Rütter et al 1995, Küpfer 2000, Job et al 2003). Sie basieren auf der Idee des keynesianischen Einkommensmultiplikators, welcher den durch eine «Geldinjektion» von aussen ausgelösten Einkommenszuwachs einer (nationalen oder regionalen) Ökonomie ausdrückt (Armstrong \& Taylor 2001).

Regionalwirtschaftliche Effekte entstehen dabei in mehreren Wirkungsrunden. Ausgehend von den (empirisch zu ermittelnden) Besucherausgaben (Gleichung 1) lässt sich der Bruttoumsatz (2) berechnen. Nach Abzug der Mehrwertsteuer ergibt sich daraus der Nettoumsatz (3). Das Einkommen der ersten Umsatzstufe $E_{1}$ umfasst die Einkommenswirkungen, die sich aus dem Nettoumsatz abzüglich der Vorleistungen ergeben (4). Die erste Umsatzstufe bildet damit den im Produktionsprozess geschaffenen Mehrwert - die Wertschöpfung - ab.

Einkommen wird aber auch über die in der Region bezogenen Vorleistungen sowie über regionale Investitionen und über die Löhne der in der ersten Wirkungsrunde profitierenden Akteure geschaffen (Abbildung 1). In sehr vielen ökonomischen Wirkungsanalysen im Tourismusbereich werden aber nur die Vorleistungseffekte berücksichtigt. Aufgrund der fehlenden statistischen Daten zu Investitionsund Lohneffekten, welche nur mit viel Aufwand selbst erhoben werden könnten, geschieht dies auch hier. Das Einkommen der zweiten Umsatzstufe $E_{2}$ ergibt sich damit aus der Wertschöpfung der in der Region bezogenen Vorleistungen (5). Diese Prozesskette liesse sich unendlich fortsetzen. Meist - so auch hier - beschränkt sich die Ermittlung der Einkommenswirkungen auf die Einkommen der ersten und zweiten Umsatzstufe (6).

Die Einkommenseffekte lassen sich auch als Beschäftigungseffekte darstellen. Hierfür müssen die gesamten Einkommenswirkungen durch die durchschnittliche Bruttowertschöpfung pro Vollzeitäquivalent (Beschäftigung umgerechnet in Vollzeitstellen) in der Region dividiert werden (7). Als Resultat ergibt sich eine fiktive Zahl von Vollzeitstelleninhabern, die über einen bestimmten Wirtschaftszweig wie zum Beispiel den «Waldtourismus» ihren Lebensunterhalt verdienen.

\section{Berechnungsgrundlagen}

Durchschnittliche Besucherausgaben für die Leistungen einer Wirtschaftsbranche pro Tag $a_{b r}$ :

$$
a_{b r}=\frac{\sum_{i=1}^{n} A_{b r, i}}{\sum_{i=1}^{n} R_{i}}
$$

wobei $A_{b r, i}$ für die über den gesamten Aufenthalt des $i$-ten Besuchers summierten Ausgaben für die Leistungen der Branche $b r$ steht, $R_{i}$ die Aufenthaltsdauer des $i$-ten Besuchers in Tagen in der Untersuchungsregion ist und $n$ die Besucherzahl im jeweiligen Untersuchungszeitraum ausdrückt. 
Bruttoumsatz $U_{b, b r}$ der Besucherausgaben nach Branchen differenziert:

$$
U_{b, b r}=B \times a_{b r}
$$

wobei $B$ die Besuchstagezahl im Untersuchungszeitraum in der interessierenden Destination innerhalb der Untersuchungsregion bezeichnet.

Nettoumsatz der Besucherausgaben $U_{n}$ :

$$
\begin{aligned}
& U_{n, b r}=\frac{U_{b, b r}}{1+M W S t_{b r}} \\
& U_{n}=\sum_{b r} U_{n, b r}
\end{aligned}
$$

wobei $U_{n, b r}$ für den branchenspezifischen Nettoumsatz und MWSt $t_{b r}$ für die branchenspezifische Umsatzsteuer stehen.

Einkommen der ersten Umsatzstufe $E_{1}$ :

$$
E_{1}=\sum_{b r} U_{n, b r}-V_{b r}=\sum_{b r} U_{n, b r} \times W_{b r}
$$

wobei $V_{b r}$ die branchenspezifische Vorleistung ausdrückt. Gleichbedeutend kann $E_{1}$ auch über die branchenspezifische Wertschöpfungsquote $w_{b r}$ berechnet werden.

Einkommen der zweiten Umsatzstufe $E_{2}$ :

$$
E_{2}=\left(U_{n}-E_{1}-V_{i m p}\right) \times w
$$

wobei $V_{\text {imp }}$ für die in die Region importierten Vorleistungen steht und $w$ die über alle Branchen gemittelte Wertschöpfungsquote bezeichnet. Aufgrund fehlender Daten wird häufig mit Annahmen gearbeitet und zum Beispiel nicht zwischen den Branchen differenziert.

Gesamte Einkommenswirkungen E:

$$
E=E_{1}+E_{2}
$$

Beschäftigungseffekt $B E$ :

$$
B E=\frac{E}{B W S_{V Z A ̈}}
$$

wobei $B W S_{V Z \ddot{A}}$ die Bruttowertschöpfung pro Vollzeitäquivalent ausdrückt. Idealerweise sollten diese regionsspezifisch sein, für die Schweiz liegen jedoch keine entsprechenden amtlichen Daten vor.

\section{Conjoint-Analyse}

Bislang wurde von den Ausgaben aller Besucher ausgegangen - unabhängig davon, ob die Besucher am Wald interessiert sind oder nicht. Für die Berechnung der regionalökonomischen Effekte soll aber nicht die Gesamtzahl der Waldbesucher be- trachtet werden, sondern die Zahl der Gäste, die sich als «waldaffin» bezeichnen lassen (Loomis \& Caughlan 2006). Die Waldaffinität kann anhand der Entscheidungskriterien der Besucher für die Wahl des Naherholungs- oder Reiseziels beurteilt werden.

In der vorliegenden Studie wird die Waldaffinität mittels einer Conjoint-Analyse bestimmt. Mit dieser Methode lassen sich Präferenzen erfassen. Erhoben werden Urteile über den jeweiligen Gesamtnutzen bestimmter Objekte oder Produkte. Davon ausgehend wird der Beitrag einzelner Produktmerkmale zum spezifischen Gesamtnutzen ermittelt. Mithilfe einer Conjoint-Analyse lässt sich demnach die relative Wichtigkeit verschiedener Produktmerkmale für die Kaufentscheidung berechnen. Zudem können die bevorzugten Merkmalsausprägungen eruiert werden. Die Beurteilung der Merkmale erfolgt bei einer Conjoint-Analyse gleichzeitig (they are considered jointly).

Der Conjoint-Analyse liegen verschiedene Annahmen zugrunde, und sie muss mehrere Anforderungen erfüllen (Backhaus et al 2003), zum Beispiel: 1. Die ausgewählten Merkmale sollten relevant, das heisst für das Urteil beziehungsweise die Auswahlentscheidung tatsächlich von Bedeutung sein. 2. Sie sollten unabhängig voneinander sein, sodass der wahrgenommene Nutzen einer Merkmalsausprägung nicht durch die Ausprägungen anderer Merkmale beeinflusst wird.

3. Es wird unterstellt, dass sich der Gesamtnutzen aus den Teilnutzen der verschiedenen Merkmale additiv zusammensetzt (Gleichung 8) und dass sich Merkmalsausprägungen demzufolge gegenseitig kompensieren lassen.

$$
y_{k}=\sum_{j=1}^{\prime} \sum_{m=1}^{M} \beta_{j, m} \times x_{j, m}
$$

wobei $y_{k}$ den geschätzten Gesamtnutzenwert für das Produkt $k$ ausdrückt und $\beta_{j, m}$ den Teilnutzen für Ausprägung $m$ von Merkmal $j$ bezeichnet. $x_{j, m}$ ist 1 , falls bei Produkt $k$ das Merkmal $j$ in Ausprägung $m$ vorliegt, ansonsten ist $x_{j, m}$ gleich 0 .

Den Befragungsteilnehmern wird meist nur eine Auswahl aller theoretisch möglichen Kombinationen von Merkmalsausprägungen zur Beurteilung vorgelegt, wobei sich die repräsentative Kombinationenauswahl über eine Statistiksoftware bestimmen lässt. Für ein solches reduziertes Design spricht, dass je nach Zahl der Merkmale und der Ausprägungen die Anzahl der möglichen Kombinationen sehr gross sein kann. Die Beurteilung der Kombinationen durch die Befragten kann unterschiedlich erfolgen. In der vorliegenden Analyse geschieht das per Rangreihung. Ausgehend von der Rangfolge lässt sich über ein statistisches Modell individuell für jeden Befragten auf die Teilnutzenwerte aller Merkmalsausprägungen und darauf aufbauend auf die Gesamtnutzenwerte aller Merkmalskombinationen sowie auf 

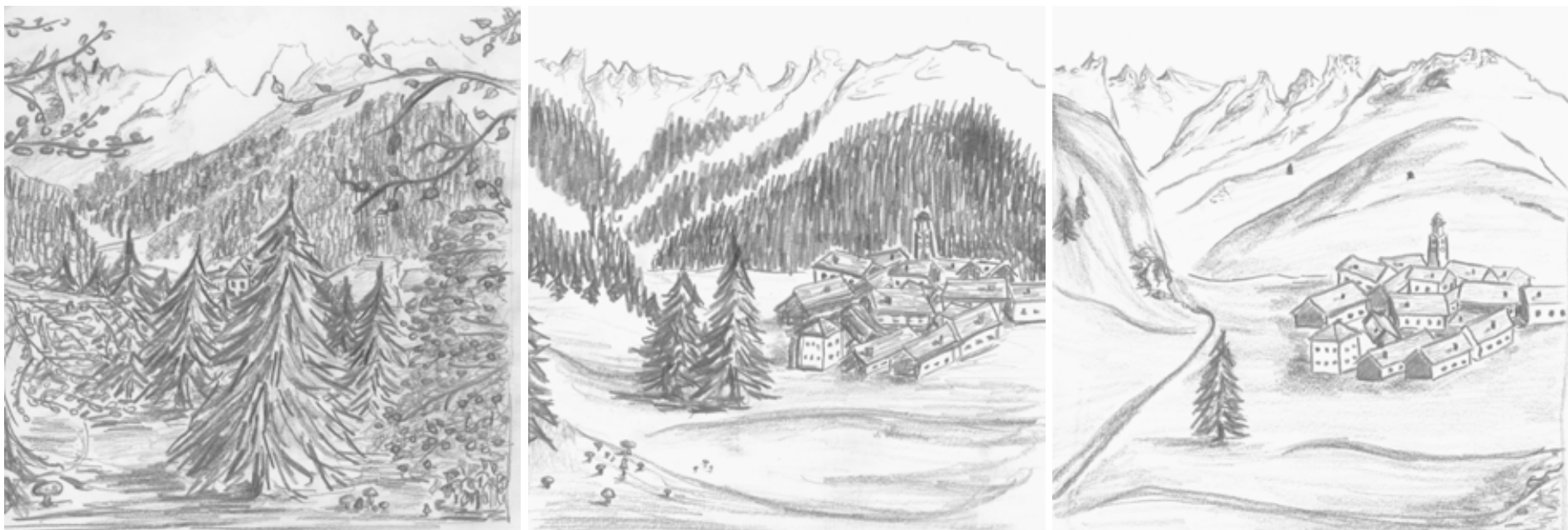

Abb 2 Landschaftsbilder der Conjoint-Analyse im Bergell. Zeichnungen: Irene Vogt

die relativen Wichtigkeiten der einzelnen Merkmale für die Beurteilung schliessen (zur mathematischen Modellierung siehe Backhaus et al 2003).

Im Rahmen der Studie waren (tages)touristische Destinationen als Ausflugs- beziehungsweise Ferienziele zu beurteilen. Die beiden Untersuchungsgebiete unterschieden sich hinsichtlich Landschaftsbild, Preisniveau, Freizeitangebot, Gastronomie- und (Para-)Hotellerieangebot. Diese Produktmerkmale können als wichtige Entscheidungskriterien für die Besucher gelten. Zu ihrer Ermittlung dienten die einschlägige Literatur und eine kurze Umfrage im Vorfeld der eigentlichen empirischen Erhebungen (mit willkürlicher Stichprobe). Die Produktmerkmale entsprechen weitgehend den oben genannten Anforderungen an die Conjoint-Analyse.

Jedes Entscheidungskriterium wies zwei oder drei Ausprägungen auf, welche den Befragungsteilnehmern grafisch und zum Teil mit erläuterndem Text präsentiert wurden. Das Landschaftsbild variierte hinsichtlich des Stellenwerts des Waldes (Abbildung 2), wobei die Orografie und die Kulturlandschaftselemente konstant gehalten wurden, das Preisniveau wechselte zwischen überdurchschnittlich teuer und relativ günstig, das Freizeitangebot unterschied sich in der touristischen Infrastruktur (primär für Outdoor-Aktivitäten), das Gastronomie- und Unterkunftsangebot war entweder relativ einfach oder sehr gepflegt. Die Befragten wurden gebeten, zehn Destinationen mit unterschiedlichen Merkmalsausprägungen (die Kombinationen waren über ein reduziertes, orthogonales Design ermittelt worden) gemäss ihren Präferenzen in eine Rangfolge zu bringen. Dabei sollten sie sich vorstellen, sie könnten die Destination für Ferien beziehungsweise für einen Ausflug im gleichen Zeitraum nochmals auswählen. Als waldaffin wurden diejenigen Besucher klassifiziert, für die das Landschaftsbild bei der Entscheidung die grösste Rolle spielte und die dabei eine waldreiche Landschaft bevorzugten (Abbildung 2 links und Mitte).

\section{Forschungsdesign}

Für die regionalökonomische Bewertung der Erholungsleistung von Wäldern sind demnach Daten notwendig zum Besuchsaufkommen in den Wäldern, zum Anteil der waldaffinen Besucher, zu den durchschnittlichen Besucherausgaben pro Tag differenziert nach Branchen, zu den Wertschöpfungsquoten und zum Anteil regionaler Vorleistungen. Aufgrund der grossen Menge an Daten, die empirisch ermittelt werden müssen, werden Wertschöpfungsstudien meist nur für ausgewählte Regionen unternommen. Auch in unserer Untersuchung wurde ein Fallstudienansatz gewählt. Dabei sollten die Wälder der beiden Untersuchungsregionen von möglichst unterschiedlichen Besuchern (Tages- versus Übernachtungsgäste) besucht werden.

Da für keine der beiden Fallstudienregionen Besuchszahlen vorlagen, wurden zwischen Juni 2008 und Mai 2009 an jeweils rund 25 Tagen parallel zu den Besucherbefragungen Zählungen durchgeführt. Als Zähl- und Befragungsstandorte wurden die wichtigsten Zugänge zu den Wäldern ausgewählt. Die Tage waren über das Jahr verteilt, um von Wetter, Wochentag und Saison abhängige Besucherzahlvariationen zu erfassen (Ploner \& Brandenburg 2003). Basierend auf den Zähldaten erfolgte die Hochrechnung der Jahresbesucherzahl auf einer Wettervariablen (gute - schlechte Witterung), einer Saisonvariablen (Sommer - Winter, Nebensaison - Hauptsaison) sowie einer Wochentagvariablen (Werktag - Wochenende, Samstag - Sonntag). Die Wetterdaten, die der Berechnung des Wetter-Dummys dienten, waren von MeteoSchweiz sowie vom Centro Meteorologico Lombardo (Italien) bezogen worden.

Parallel zu den Zählungen wurde eine Zufallsauswahl der Waldgäste mittels eines standardisierten Fragebogens mündlich befragt. Im Sihlwald wurden 566 Interviews geführt (79\% der angefragten Passanten willigten in die Befragung ein), im Bergell 367 (bei einer Antwortquote von 70\%). Hauptinhalt der auf Deutsch oder Italienisch durchgeführten In- 
terviews waren Fragen zum Ausgabeverhalten sowie die Conjoint-Analyse. Die Conjoint-Daten konnten von 263 Befragten im Sihlwald und 161 Teilnehmern im Bergell ausgewertet werden. Ob die umfangreiche und geistig fordernde Conjoint-Aufgabe (vollständig) gelöst wurde oder nicht, lässt sich mit keiner anderen Antwort der Befragten in einen signifikanten Zusammenhang bringen. Wir gehen deshalb davon aus, dass die Stichprobe der Conjoint-Aufgabe in etwa der Befragungsstichprobe entspricht.

Wertschöpfungsquoten $\left(w_{b r}, w\right)$ und der Anteil regionaler Vorleistungen $\left(V_{\text {imp }}\right)$ wurden nicht empirisch ermittelt. Idealerweise müssten regionsspezifische Quoten verwendet werden, für die Schweiz liegen jedoch keine entsprechenden Daten vor, sodass auf nationale Werte ausgewichen wurde. ${ }^{1}$ Zur Erfassung des Einkommens der zweiten Umsatzstufe $\left(E_{2}\right)$ wurde in Anlehnung an Küpfer (2000) für den Anteil der in die Region importierten Vorleistungen $\left(V_{\text {imp }}\right)$ branchenübergreifend ein Wert von 50\% angenommen. Auf 50\% beläuft sich auch die über alle Branchen gemittelte Wertschöpfungsquote (w). Auch für die Bruttowertschöpfung pro Vollzeitäquivalent $\left(B W S_{V Z \ddot{A}}\right)$ wurden gesamtschweizerische Werte beigezogen $^{1,2}$ (in den BFS-Beschäftigungsstatistiken fehlen Angaben zur Landwirtschaft und zu persönlichen Dienstleistungen, weshalb sie nicht ins Mittel für alle Wirtschaftszweige einfliessen).

\section{Untersuchungsregionen}

Die Untersuchungen fanden im Sihlwald und im Bergell statt. Der Sihlwald (Abbildung 3) liegt zwischen Zürich und Zug und bildet mit den angrenzenden Wäldern die grösste zusammenhängende Waldfläche im Schweizer Mittelland. Der Sihlwald

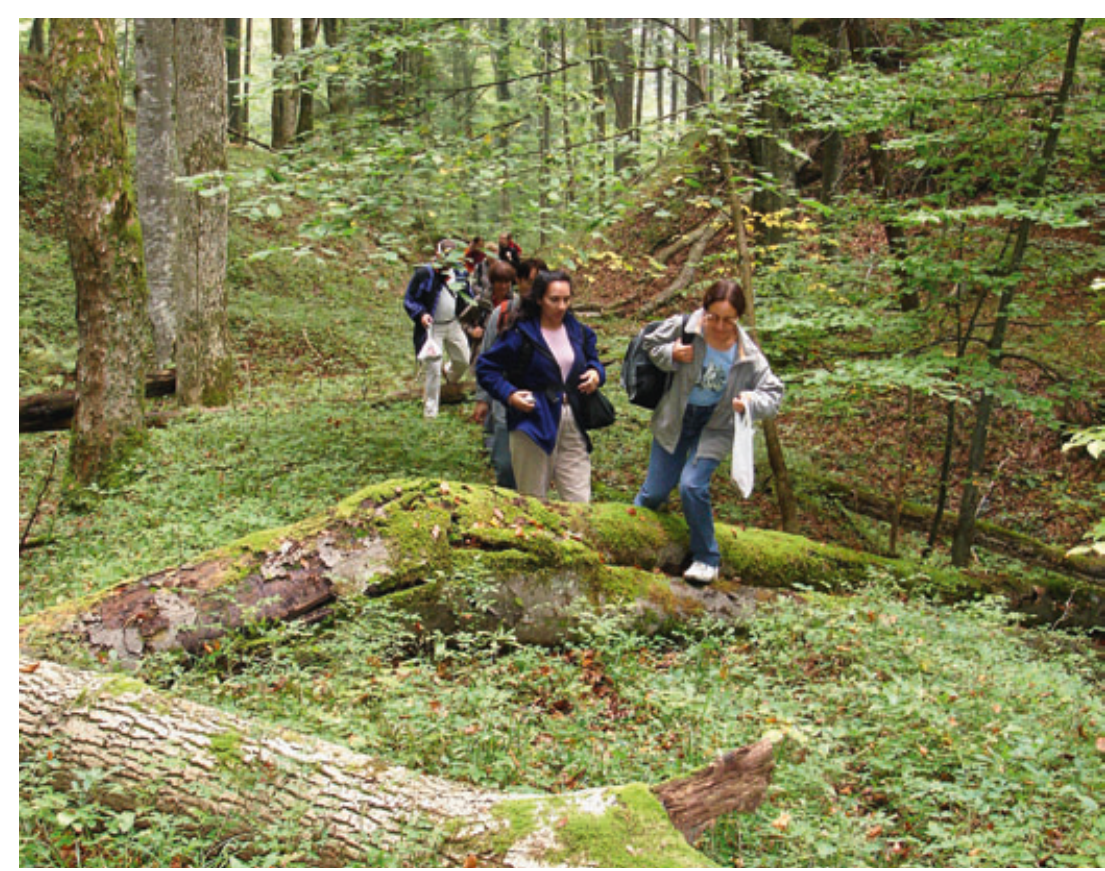

Abb 3 Mehr als die Hälfte der Gäste im Sihlwald können als «waldaffin» bezeichnet werden, das heisst, sie bevorzugen Landschaftsbilder mit viel Wald und messen der Landschaft bei der Destinationswahl einen sehr hohen Stellenwert bei. Foto: Wildnispark Zürich bedeckt eine Fläche von rund 1000 Hektaren und ist seit 2009 als Naturerlebnispark gemäss Bundesgesetz vom 1. Juli 1966 über den Natur- und Heimatschutz (NHG, SR 451) anerkannt. Seit 2000 wird der Wald nicht mehr bewirtschaftet. Im Vordergrund stehen der Prozessschutz, die Ermöglichung von Naturerlebnissen sowie die Umweltbildung. Die Untersuchungsregion der Wertschöpfungsanalyse umfasste alle Gemeinden mit Flächenanteilen am Sihlwald, namentlich Horgen, Oberrieden, Thalwil, Langnau am Albis, Hausen am Albis und Hirzel. Bei diesen handelt es sich um dicht besiedelte Gemeinden mit typisch suburbaner Wirtschaftsstruktur. In die Untersuchung einbezogen wurden nur Personen, welche sich im Waldgebiet selbst und längs der Sihl aufhielten, nicht aber Gäste, die zum Beispiel ausschliesslich das Besucherzentrum aufsuchten.

Das Bergell, ein Alpental im südlichen Graubünden, liegt zwischen Malojapass und der italienischen Grenze. Die meisten Wälder liegen an sehr aufwendig zu bewirtschaftenden Steillagen und werden kaum mehr gewinnorientiert forstwirtschaftlich genutzt. Im Vordergrund steht die Aufrechterhaltung der Schutzleistung der Wälder. Aufgrund des Rückgangs der Landwirtschaft nahm die Bewaldung in den vergangenen 60 Jahren um etwa 30\% auf heute rund 5200 Hektaren zu (Amt für Wald 2006, Amt für Raumentwicklung Graubünden 2010). Die Untersuchungsregion umfasst die 2010 zur Gemeinde Bregaglia (Bergell) fusionierten früheren Gemeinden Bondo, Castasegna, Soglio, Stampa und Vicosoprano (Abbildung 4). Die gesamte Region ist dünn besiedelt und verzeichnete im vergangenen Jahrzehnt einen Bevölkerungsrückgang. Die Beschäftigungsstruktur ist vom primären Sektor, vom Baugewerbe, vom Tourismus und von öffentlichen Diensten geprägt. ${ }^{3}$ Befragt wurden alle Erholungsuchenden, die sich in den Wäldern aufhielten.

\section{Regionalökonomische Bewertung}

\section{Besuchszahlen und Besuchertypen}

Die auf den Zählungen basierenden Hochrechnungen ergeben für den Sihlwald rund 137000 Besuchstage im Erhebungsjahr 2008/09. Daraus resultiert eine Besucherdichte von rund 137 Besuchstagen pro Jahr und Hektare. Der Sihlwald wird fast ausschliesslich von Tagestouristen (60\%) und Naherholern aus den Sihlwald-Gemeinden (38\%) besucht. Gäste, die hier ihre Ferien verbringen, machen nur knapp 2\% der Waldbesucher aus.

\footnotetext{
1 BFS (2010) Volkswirtschaftliche Gesamtrechnung. Neuchâtel: Bundesamt Statistik. Internes Dokument.

2 BFS (2010) Beschäftigungsstatistik. Neuchâtel: Bundesamt Statistik. Internes Dokument.

3 BFS (2009) Betriebszählung 2008. Neuchâtel: Bundesamt Statistik. Internes Dokument.
} 
Abb 4 Vicosoprano ist eine der fünf früheren Gemeinden im Bergell. Heute gehört Vicosoprano zur Gemeinde Bregaglia. Foto: Ruth Wolf

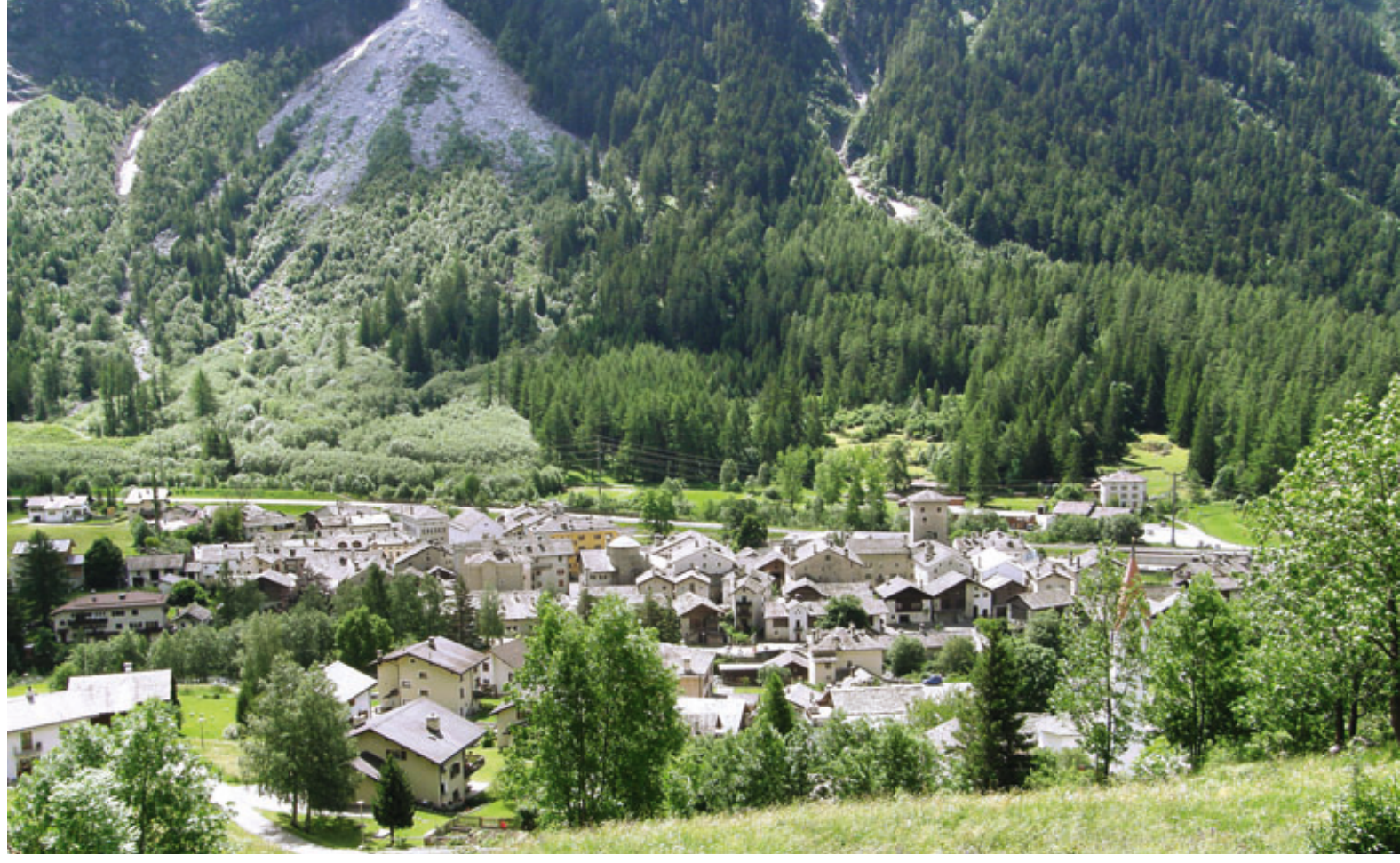

Im Bergell präsentiert sich die Situation umgekehrt. Rund 52\% der Waldgäste sind ferienhalber im Bergell. 48\% können als Tagesgäste bezeichnet werden. Typischerweise reisen sie aus Italien oder ihrem Ferienort im Engadin an (weniger als 5\% kommen vom Wohnort im Bergell). Für die Bergeller Wälder ermittelten wir einen Wert von rund 86000 Besuchstagen und damit eine Besucherdichte von 17 Besuchstagen pro Jahr und Hektare.

\section{Anteil der waldaffinen Besucher}

Im Sihlwald ist das Landschaftsbild das mit Abstand wichtigste Entscheidungskriterium für die Destinationswahl. Zwischen den Befragten, welche die an einer anderen Stelle im Fragebogen abgefragte Bedeutung der Erreichbarkeit als hoch einstuften, und denen, für welche die Nähe zum Wald keine Rolle für die Destinationswahl spielte, gibt es keine signifikanten Unterschiede. Knapp 54\% der Waldgäste werten die Landschaft als das wichtigste der vier Produktmerkmale, wobei 52\% als waldaffin definiert werden können, das heisst, sie priorisieren die Landschaft und ziehen dabei die Landschaftsbilder vor, in denen der Wald ein deutliches Element des Landschaftsbildes darstellt. Die Ergebnisse sind hochsignifikant ( $p=0.000$, Pearson $r=0.998$, Kendall's Tau $=1.000$ ). Es gibt einen Zusammenhang zwischen Waldaffinität der Besucher und Besuchertyp. Die Tagesgäste sind tendenziell waldaffiner als die Naherholer ( $\mathrm{p}=0.05$, Cramer $\mathrm{V}=0.151$ ).

Für das Bergell zeigen die Ergebnisse der Conjoint-Analyse ein ähnliches Gewicht der vier Entscheidungskriterien. Für 39\% der Waldgäste hat das Landschaftsbild bei der Wahl der Destination den höchsten Stellenwert. Dabei können insgesamt 35\% als waldaffin gelten. Auch im Bergell sind die Resultate hochsignifikant $(\mathrm{p}=0.000$, Pearson $\mathrm{r}=0.999$,
Kendall's Tau $=1.000$ ). Hingegen konnte kein statistisch signifikanter Zusammenhang zwischen Waldaffinität und Besuchertyp gefunden werden.

\section{Regionalökonomische Effekte}

Demzufolge können im Sihlwald 52\% und im Bergell 35\% der regionalökonomischen Effekte, die durch alle Waldgäste ausgelöst werden, der Erholungsleistung von Wäldern zugerechnet werden. Im Sihlwald geben die Tagesgäste und Naherholer im Zusammenhang mit ihrem Waldbesuch im Mittel CHF 10.- pro Tag aus. Bei den Feriengästen, die meistens privat übernachten, belaufen sich die Ausgaben pro Besucher und Tag auf CHF 24.-. Im Bergell geben die Tagestouristen im Mittel CHF 38.- aus. Die Ausgaben der Feriengäste, die meist in Hotels, Pensionen und Ferienwohnungen logieren, betragen CHF 108.- pro Tag (Tabelle 1). In beiden Untersuchungsregionen sind die Tagesausgaben der Besucher statistisch unabhängig von der Waldaffinität. Der durch die waldaffinen Gäste ausgelöste Bruttoumsatz beläuft sich im Sihlwald auf CHF 0.73 Mio., im Bergell auf CHF 2.23 Mio., der Nettoumsatz auf CHF 0.68 Mio. beziehungsweise CHF 2.11 Mio.

Tabelle 1 zeigt auch die Einkommen der ersten und zweiten Umsatzstufe. Insgesamt bewirkt die Erholungsleistung des Sihlwalds Einkommenseffekte in der Höhe von CHF 0.43 Mio. Die Einkommenswirkungen der Bergeller Wälder lassen sich auf CHF 1.36 Mio. schätzen. Die Ökosystemleistung Erholung generiert damit in der Sihlwald-Region drei beziehungsweise sieben Vollzeitarbeitsplätze, im Bergell zehn beziehungsweise 22 Vollzeitäquivalente (je nachdem, ob die Bruttowertschöpfung pro Vollzeitäquivalent für alle Sektoren oder nur für das Gastgewerbe betrachtet wird). Die gesamten Einkommenswirkungen, die von allen (nicht nur von den 


\begin{tabular}{|l|l|c|c|}
\hline Regionalökonomische Effekte & & Sihlwald & Bergell \\
\hline $\begin{array}{l}\text { Besuche in der } \\
\text { Untersuchungsregion }\end{array}$ & waldaffine Gäste & 71000 & 30000 \\
\hline Durchschnittliche Besucheraus- & total Gäste & 137000 & 86000 \\
\hline gaben pro Tag (CHF) & durch Tagesgäste & 10.00 & 38.05 \\
\hline Bruttoumsatz (CHF) & durch Feriengäste & 23.55 & 107.80 \\
\hline Nettoumsatz (CHF) & & 0.73 Mio. & 2.23 Mio. \\
\hline $\begin{array}{l}\text { Einkommen der ersten Umsatz- } \\
\text { stufe (CHF) }\end{array}$ & 0.68 Mio. & 2.11 Mio. \\
\hline $\begin{array}{l}\text { Einkommen der zweiten Um- } \\
\text { satzstufe (CHF) }\end{array}$ & 0.35 Mio. & 1.11 Mio. \\
\hline $\begin{array}{l}\text { Gesamte Einkommenswirkungen } \\
\text { (CHF) }\end{array}$ & & 0.08 Mio. & 0.25 Mio. \\
\hline $\begin{array}{l}\text { Beschäftigungseffekte } \\
\text { (in Vollzeitäquivalenten) }\end{array}$ & in allen Sektoren & 3 & 10 \\
\hline & im Gastgewerbe & 7 & 22 \\
\hline
\end{tabular}

Tab 1 Regionalökonomische Effekte der Erholungsleistung in den beiden Untersuchungsregionen Sihlwald und Bergell, welche von den waldaffinen Gästen ausgehen.

waldaffinen) Waldgästen ausgehen, belaufen sich im Bergell auf CHF 3.91 Mio., im Sihlwald beträgt der Beitrag aller Waldbesucher zum Bruttoinlandprodukt der Region CHF 0.83 Mio.

\section{Diskussion der Ergebnisse}

Gemäss dem Schweizerischen Landesforstinventar (LFI, Brändli 2010) ist die Besucherdichte im Sihlwald gering und entspricht der Erholungsnutzung von $41 \%$ der Waldfläche im Schweizer Mittelland. Auf $33 \%$ der Waldfläche im Mittelland ist die Besucherdichte höher. Der Schutzgebietsstatus des Sihlwalds und seine Lage in der Agglomeration Zürich führen also nicht zu einer erhöhten Besucherzahl im Waldgebiet. Eine mögliche Erklärung dafür ist, dass sich Gäste, welche vor allem an der Unterschutzstellung interessiert sind, primär im Besucherzentrum und nicht im Wald selbst aufhalten. Diese Gäste wurden in der Untersuchung nicht berücksichtigt. Zudem kann davon ausgegangen werden, dass der Sihlwald für Naherholungsuchende aus Zürich zu weit abseits gelegen und für Tagesausflügler aufgrund seiner Ähnlichkeit mit den Zürcher Stadtwäldern zu wenig aussergewöhnlich ist.

Im Bergell gilt die Intensität der Erholungsnutzung laut LFI als sehr gering. Die Besucherdichte ist in den Südalpen auf 27\% der Waldfläche höher und auf 54\% noch niedriger (Brändli 2010). Die von uns hochgerechnete Besuchszahl erscheint bei einem Vergleich mit amtlichen Tourismusstatistikzahlen als plausibler Wert. 16000 Besuche sind durch Gäste ausgelöst, die in Hotels logieren (die anderen Feriengäste übernachten in der Parahotellerie). Nimmt man die Tourismuszahlen der Wintermonate der früheren Gemeinde Stampa mit dem landschaftlich zum Engadin zugehörigen Wintersportort Maloja aus, so verzeichnete die Hotellerie im Bergell im Un- tersuchungszeitraum rund 12800 Ankünfte und 40200 Logiernächte. ${ }^{4}$ Jeder Gast besucht demnach bei einer Aufenthaltsdauer von etwas mehr als drei Tagen im Mittel 1.25-mal einen Wald im Bergell.

Die im Vergleich zum Bergell deutlich höhere Waldaffinität der Sihlwaldgäste lässt sich mit der Besucherstruktur erklären. Das bei der Naherholung oder bei einem Tagesausflug gesuchte Erlebnis setzt sich aus weniger Leistungen zusammen als in den Ferien. Im Sihlwald hat zum Beispiel das Gastronomieangebot, das nur 10\% der Entscheidungen ausmacht, eine geringe relative Bedeutung, was nicht erstaunt. Es ist naheliegend, dass die meisten Naherholer nicht an einem Gastronomieangebot interessiert sind. Entsprechend erhält das Produktmerkmal Landschaft, gefolgt vom Freizeitangebot, ein grösseres Gewicht. Die in den beiden Untersuchungsregionen stark unterschiedliche Besucherstruktur führt auch zu den erheblichen Differenzen bei den Ausgaben. Im Sihlwald konsumieren die Besucher kaum kostenpflichtige Leistungen. Dementsprechend gering ist der Beitrag zur regionalen Wertschöpfung. Nach unseren Kenntnissen liegen für die Schweiz und das benachbarte Ausland keine vergleichbaren Zahlen zum Ausgabeverhalten von Gästen von Naherholungswäldern vor. Der Wert der Freizeitnutzung von Wäldern wird häufig als gesellschaftlicher Wert bemessen, beispielsweise über Zahlungsbereitschaftsanalysen. Bernath et al (2006) eruieren eine Zahlungsbereitschaft der Zürcher Stadtbevölkerung in der Höhe von CHF 112.- für eine Jahreseintrittskarte für die Wälder auf dem Gebiet der Stadt Zürich. Die Erholungsleistung pro Hektare Stadtwald hat damit einen Wert von CHF 1300.-. Gemäss unseren Berechnungen für den Sihlwald beträgt der regionalökonomische Wert pro Hektare CHF 430.-, wenn man nur die waldaffinen Gäste berücksichtigt, und CHF 830.- bei Einbezug aller Waldgäste. Übernimmt man die für die Stadtzürcher Wälder berechneten Werte, findet sich somit nur ein Teil der gesellschaftlichen Wertschätzung in regionalen Wirtschaftskreisläufen wieder.

Die ermittelten mittleren Ausgaben pro Feriengast im Bergell sind vergleichbar mit den 1998 erhobenen Ausgabenwerten in der Schweizer Nationalparkregion in Höhe von CHF 88.- pro Übernachtungsgast (Küpfer 2000). Die Ausgaben von Tagesgästen waren in dieser Studie nicht erhoben worden. Die Bergeller Werte liegen jedoch deutlich höher als die Besucherausgaben im Berner Oberland. Im Simmental geben Übernachtungsgäste in der Sommersaison pro Tag und Kopf im Mittel CHF 67.50 aus, im Diemtigtal CHF 36.05 (Mayer et al 2009), im Bergell hingegen CHF 107.80. Die Unterschiede sind bei den Unterkunftskategorien zu suchen: Im

\footnotetext{
$\overline{4 \text { BFS }(2009}, 2010)$ Beherbergungsstatistik. Neuchâtel: Bundesamt Statistik. Interne Dokumente.
} 
Berner Oberland übernachtet ein Grossteil der Gäste in der günstigeren Parahotellerie. Auch die Ausgaben der Bergeller Tagestouristen sind höher als diejenigen im Berner Oberland. Hier dürften eine Mautgebühr für eine stark frequentierte Strasse in ein Seitental sowie die rege Nutzung einer Seilbahn die Differenz erklären. Die regionalökonomische Bedeutung der Freizeitnutzung der Wälder im Bergell ist erheblich, auch wenn sie bezogen auf die Fläche mit CHF 261.- pro Hektare (waldaffine Gäste) beziehungsweise CHF 750.- pro Hektare (alle Waldgäste) geringer ist als im Sihlwald. Ohne die frühere Gemeinde Stampa gibt es im Bergell 95 Vollzeitäquivalente im Bereich Beherbergung und Gastronomie. Rechnet man die Beschäftigungseffekte der Erholungsleistung von Wäldern allein der Gastronomie zu, lassen sich 23\% der Arbeitsplätze im Tourismus auf die Wälder zurück-führen (mit Stampa 13\%).

\section{Schlussfolgerungen}

Generalisierungen aus zwei Fallstudien sind unzulässig. Die abschliessenden Überlegungen sind daher eher als Thesen zu verstehen. Der regionalökonomische Wert typischer «Naherholungswälder» in suburbanen, ländlichen Räumen ist tendenziell vernachlässigbar. Grund dafür sind die Besucherstruktur und die meist wenigen Möglichkeiten, in den Wäldern beziehungsweise im Umfeld der Wälder Geld auszugeben. In Ferienregionen, vor allem im peripheren, ländlichen Raum, bringt die Freizeitnutzung der Wälder der lokalen Wirtschaft weit mehr. Übernachtungsgäste geben nicht nur für die Unterkunft mehr aus, sondern auch in der Gastronomie oder im Einzelhandel. Wie hoch die Ausgaben sind, hängt letztlich immer auch vom Spektrum und von der Qualität touristischer Leistungen im Umfeld der Wälder ab. Trotzdem dürfte gelten, dass in Naherholungswäldern die Waldaffinität weit höher ist als in Ferienregionen.

Soll die Freizeitnutzung von Wäldern eine grössere regionalwirtschaftliche Bedeutung in ländlichen Räumen entfalten, müssen touristische Angebote im Umfeld der Wälder geschaffen oder aufgewertet werden. Eine stärkere Inwertsetzung von Wäldern könnte auch dadurch erfolgen, dass man in den Wäldern selbst private Güter rund um Freizeit und Erholung schafft und anbietet, wie zum Beispiel Seilparks, Baumwipfelpfade oder geführte Waldexkursionen. So könnten auch forstwirtschaftliche Betriebe aus der Erholungsfunktion von Wäldern einen Nutzen ziehen. Wenn an solchen Angeboten jedoch kein Interesse besteht (wie für stadtnahe Naherholungswälder wie den Sihlwald zu vermuten), sind Einnahmen über freiwillige Spenden aus Kurtaxen oder Landschaftspflegebeiträgen denkbar. Regionalökonomische Effekte würden auch dann ausgelöst, wenn die öffentliche Hand über Leistungsvereinbarungen die Waldeigentümer für ihre Erholungsvorsorgeleistungen, zum Beispiel für den Wegeunterhalt oder für das Freihalten von Sichtachsen, entlohnen würde. Das Waldgesetz ermöglicht dies eigentlich.

Aus den Ergebnissen und den Schlussfolgerungen ist der Wunsch nach weiteren regionalen Fallstudien abzuleiten, um die Ergebnisse dieses Beitrags validieren und verschiedene Wälder und Regionen hinsichtlich der regionalökonomischen Effekte ihrer Erholungsleistungen vergleichen zu können. Darüber hinaus sollte sich die Forschung zukünftig intensiver mit der Frage beschäftigen, welche institutionellen Rahmenbedingungen die Inwertsetzung des Waldes für Freizeit und Erholung positiv oder negativ beeinflussen. Das Bundesamt für Umwelt (Bafu) hat dazu wertvolle Grundlagen erarbeiten lassen (Bernasconi \& Schroff 2008, Roschewitz \& Holthausen 2007). Hier gilt es anzuknüpfen und die Forschung stärker auf Entscheidungsprozesse und Koordinationsformen rund um die Freizeitnutzung von Wäldern auszurichten. Wenn die Forschung verstärkt eine Governance-Perspektive einnimmt (Howlett et al 2009) und so Institutionen intensiver betrachtet, ist es auch möglich, die in vielen Fällen ungelöste Frage der Abgeltung von Leistungen der Waldeigentümer und Forstbetriebe zu regeln.

Abschliessend erwähnt werden soll die zweite Befragung der Schweizer Bevölkerung zum Wald (Wald-Monitoring soziokulturell, WaMos 2), welche 2010 von der WSL im Auftrag des Bafu durchgeführt wurde und die Befragung aus dem Jahr 1997 (Buwal 2005) wiederholt. Erste Ergebnisse werden Ende 2011 veröffentlicht. Sie dürften ein besseres Verständnis der Waldaffinität und ihrer Einflussfaktoren ermöglichen und damit eine Inwertsetzung der Erholungsleistungen von Wäldern, die nachfrageorientierter und zielgruppenspezifischer ist als bisher.

Eingereicht: 1. März 2011, akzeptiert (mit Review): 28. Juli 2011

\section{Dank}

Wir danken dem Staatssekretariat für Bildung und Forschung (SBF) für die Finanzierung des Projekts im Rahmen der COST-Aktion E45 «European Forest Externalities».

\section{Literatur}

AMT FÜR RAUMENTWICKLUNG GRAUBÜNDEN (2010) Raumbeobachtung Kanton Graubünden. www.gr.ch/DE/institutionen/verwaltung/dvs/are/Documents/Raumbeobachtung.pdf (5.2.2011)

AMT FÜR WALD (2006) Bregaglia/Bergell 1942-2003. Sviluppo della superficie boschiva / Waldflächenentwicklung. Zuoz: Amt Wald (Südbünden). CD-ROM.

ARMSTRONG H, TAYLOR J (2001) Regional economics and policy. Oxford: Malden. 448 p. 
BACKHAUS K, ERICHSON B, PLINKE W, WEIBER R (2003) Multivariate Analysemethoden. Berlin: Springer. $818 \mathrm{p}$.

BERNASCONI A, SCHROFF U (2008) Freizeit und Erholung im Wald. Grundlagen, Instrumente, Beispiele. Bern: Bundesamt Umwelt, Umwelt-Wissen 0819. 69 p.

BERNATH K, ROSCHEWITZ A, STUDHALTER S (2006) Die Wälder der Stadt Zürich als Erholungsraum. Birmensdorf: Eidgenöss Forsch.anstalt Wald Schnee Landschaft. 43 p.

BRÄNDLI UB, EDITOR (2010) Schweizerisches Landesforstinventar. Ergebnisse der dritten Erhebung 2004-2006. Birmensdorf: Eidgenöss Forsch.anstalt Wald Schnee Landschaft. $312 \mathrm{p}$.

BUWAL (2005) Gesellschaftliche Ansprüche an den Schweizer Wald. Ergebnisse einer Meinungsumfrage des Buwal 1998. Bern: Bundesamt Umwelt Wald Landschaft. 152 p.

HOWLETT M, RAYNER J, TOLLEFSON C (2009) From government to governance in forest planning? Lessons from the case of the British Columbia Great Bear Rainforest Initiative. For Policy Econ 11: 383-391.

JOB H, METZLER D, VOGT L (2003) Inwertsetzung alpiner Nationalparks. Eine regionalwirtschaftliche Analyse des Tourismus im Alpenpark Berchtesgaden. Kallmünz: Lassleben. $164 \mathrm{p}$.

KÜPFER I (2000) Die regionalwirtschaftliche Bedeutung des Nationalparktourismus untersucht am Beispiel des Schweizerischen Nationalparks. Zernez: Forsch.komm Schweiz Nationalpark. 143 p.

LOOMIS J, CAUGHLAN L (2006) The importance of adjusting for trip purpose in regional economic analyses of tourist destinations. Tour Econ 12: 33-43.

\section{Zur Freizeitnutzung von Schweizer Wäldern: ihre Bedeutung für die regionale Ökonomie}

Wälder erfüllen Schutz-, Nutz- und Wohlfahrtsfunktionen. Wird der Wald nicht isoliert, sondern als Teil der Landschaft oder als Teil einer Region betrachtet, werden zudem die positiven Effekte für die regionale Wohlfahrt deutlich. Am Beispiel des Sihlwalds als eines typischen Naherholungsgebiets und des Bergells als naturtouristischer Ferienregion wird die regionalökonomische Bedeutung der Freizeitnutzung von Schweizer Wäldern untersucht. Mittels Conjoint-Analyse wird die relative Wichtigkeit des Waldes für den Waldbesuch ermittelt, um die Waldaffinität der Besucher beurteilen zu können. Mittels Wertschöpfungsanalyse werden die regionalen Wertschöpfungs- und Beschäftigungseffekte der Freizeitnutzung im Wald erhoben. Naherholungswälder haben eine eher geringe ökonomische Wirkung: Die Besucher weisen zwar eine relativ hohe Waldaffinität auf, tätigen aber nur wenige Ausgaben in der Region. In Ferienregionen verhält es sich umgekehrt: Hier spielt der Wald als solcher eine geringere Rolle für die Destinationsentscheidung, während die Regionalökonomie durch höhere Konsumausgaben stärker gefördert wird. Die Höhe der Gästeausgaben hängt vor allem vom Spektrum und von der Qualität touristischer Leistungen im Umfeld der Wälder ab. Um die regionalwirtschaftliche Bedeutung der Freizeitnutzung von Wäldern zu steigern, müssten touristische Angebote im Umfeld der Wälder geschaffen oder aufgewertet werden. Eine Möglichkeit bestünde auch darin, private Güter im Bereich Freizeit und Erholung zu schaffen, damit auch forstwirtschaftliche Betriebe aus der Erholungsfunktion von Wäldern einen Nutzen ziehen können.
MANKIW NG (2004) Grundzüge der Volkswirtschaftslehre. Stuttgart: Schäffer-Poeschel. 911 p.

MAYER M ET AL (2009) Wirtschaftliche Bedeutung des naturnahen Tourismus im Simmental und Diemtigtal. Regionalökonomische Effekte und Erfolgsfaktoren. Birmensdorf: Eidgenöss Forsch.anstalt Wald Schnee Landschaft. $104 \mathrm{p}$. MAYER M, MÜLLER M, WOLTERING M, ARNEGGER J, JOB H (2010) The economic impact of tourism in six German national parks. Landsc Urban Plan 97: 73-82.

METZLER D (2007) Regionalwirtschaftliche Effekte von Freizeitgrosseinrichtungen. Kallmünz: Lassleben. 155 p.

MILLENNIUM ECOSYSTEM ASSESSMENT (2005) Ecosystems and human well-being: Synthesis. Washington: Island Press. $155 \mathrm{p}$.

OLSCHEWSKI R, ELSASSER P (2010) Mehr als «Habitat» oder «Dienstleister»? Der Wald als Komponente eines Landschaftssystems. Schweiz Z Forstwes 161: 334-340. doi: 10.3188/szf.2010.0334

PLONER A, BRANDENBURG C (2003) Modelling visitor attendance levels subject to day of the week and weather: a comparison between linear regression models and regression trees. J Nat Conserv 11: 297-308.

ROSCHEWITZ A, HOLTHAUSEN N (2007) Wald in Wert setzen für Freizeit und Erholung. Situationsanalyse. Bern: Bundesamt Umwelt, Umwelt-Wissen 0716. 39 p.

RÜTTER H, MÜLLER H, GUHL D, STETTLER J (1995) Tourismus im Kanton Bern: Wertschöpfungsstudie. Bern: Univ Bern, Berner Studien Freizeit Tourismus 34. 261 p.

\section{Utilisation récréative des forêts suisses: son importance pour l'économie régionale}

Les forêts remplissent des fonctions protectrice, sociale et économique. Si elles ne sont pas prises isolément mais considérées comme parties intégrantes d'un paysage ou d'une région, leurs effets positifs sur l'économie régionale se manifestent également. L'importance que revêt l'utilisation récréative des forêts suisses pour l'économie régionale est examinée à partir de deux exemples: celui du Sihlwald, aire typique de loisirs de proximité, et celui du Bergell, région de vacances dédiée au tourisme vert. Grâce à une analyse conjointe, on détermine l'importance relative de la forêt dans le choix d'une visite en forêt afin de pouvoir évaluer l'affinité des visiteurs envers elle. Une analyse de création de valeur permet alors d'inventorier les effets au niveau régional de l'utilisation récréative de la forêt sur la création de valeur et sur l'emploi. Les forêts de loisirs de proximité ont un impact économique plutôt faible: les visiteurs présentent certes une affinité relativement élevée envers ces forêts, mais ils n'effectuent que de faibles dépenses dans la région. Dans les régions de vacances, la situation s'inverse: la forêt en tant que telle influence moins le choix de la destination, mais en parallèle l'économie régionale est plus fortement stimulée du fait de dépenses de consommation supérieures. Leur niveau dépend avant tout de la gamme et de la qualité des prestations touristiques offertes en milieu forestier. Afin d'accroître l'importance de l'utilisation récréative des forêts dans l'économie régionale, il faudrait créer ou revaloriser des offres touristiques en milieu forestier. Autre possibilité: créer aussi des biens privés dans le secteur des loisirs et de la détente pour que les entreprises d'exploitation forestière puissent elles aussi tirer bénéfice de cette fonction récréative. 